Review Paper:

\title{
Non-Pharmacological Breathlessness Management in Older Patients: A Review Study
}

\author{
Yodang $^{1^{*} \mathrm{C}}$ \\ 1. School of Nursing, Faculty of Science and Technology, Universitas Sembilanbelas November Kolaka, Kolaka, Indonesia.
}

\begin{tabular}{|c|c|}
\hline $\begin{array}{l}\text { Use vour device to scan } \\
\text { and read the article online }\end{array}$ & dteation: Yodang, 2019. Non-pharmacological Breathlessness Management in Older Patients: A Review Study. Journal of \\
\hline 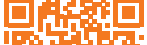 & Client-Centered Nursing Care, 5(1), pp. 1-14. http://dx.doi.org/10.32598/jccnc.5.1.1 \\
\hline 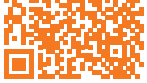 & def" http://dx.doi.org/10.32598/jcenc.5.1.1 \\
\hline
\end{tabular}

\section{(C) 03}

Article info:

Received: 10 Aug 2018

Accepted: 23 Nov 2018

Published: 01 Feb 2019

Keywords:

Aging, Cognitive therapy,

Dyspnea, Pulmonary

disease, Respiratory

muscles, Yoga

\begin{abstract}
A B S T RA C T
Background: Breathlessness is a multifactorial problem caused by multiple pathways and thus lends itself to a range of interventions, each targeting a specific mechanism. For a start, the aging process affects respiratory function and decreases lung elasticity and respiratory muscle strength, which results in forced vital capacity reduction. Moreover, many conditions, such as chronic obstructive pulmonary disease, can cause breathlessness. There is no single intervention to relieve breathlessness, particularly when we are dealing with chronic refractory breathlessness. This study aimed at conducting a review of studies on non-pharmacological breathlessness management in older patients.
\end{abstract}

Methods: This review study used the (Preferred Reporting Items for Systematic Reviews and Metaanalyses) PRISMA protocol to select appropriate resources. Two journal databases of CINAHL and MEDLINE were searched in this regard. The inclusion criteria were as follows: original articles available in full-text, peer-reviewed articles written in English from 2008 to 2018, articles focus on non-pharmacological interventions with samples of the older people or patients ( $\geq 65$ years old) complaining of dyspnea or breathlessness.

Results: There were five articles which met all the above criteria. They used non-pharmacological treatments of (Transcutaneous Electrical Nerve Simulation on acupoints) Acu-TENS, cognitive behavior therapy, inspiratory muscle training, and yoga. Most of these interventions had been applied in older patients with Chronic Obstructive Pulmonary Disease (COPD), and then patients with thoracic malignancy and lung cancer in that order.

Conclusion: Based on the results, these non-pharmacological treatments show potential benefits in improving breathlessness for older patients. However, issues such as characteristic of the sample, including co-morbidities and smoking history, and the size of the sample should be considered.

\footnotetext{
* Corresponding Author:

Yodang, PhD.

Address: Department of Nursing, School of Science and Technology, Universitas Sembilanbelas November Kolaka, Kolaka, Indonesia.

Tel: $+62(822) 91410745$

E-mail: yodangrsbgkolaka@gmail.com
} 


\section{Highlights}

- Dyspnea is common in older people, and one of its causes include the aging process.

- There was no single intervention to relieve breathlessness, and holistic intervention such as non-medical approaches is needed to provide incremental relief.

- Transcutaneous electrical nerve stimulation over acupoints, cognitive behavior therapy, inspiratory muscle training, and yoga have shown potential benefits in alleviating breathlessness among older patients.

\section{Plain Language Summary}

This is a review study about non-pharmacological treatments of breathlessness or shortness of breath management in older patients. Breathlessness is a multifactorial problem, and thus, interventions in the specific mechanisms can relieve it. Besides the effect of the aging process, many conditions can cause breathlessness such as chronic obstructive pulmonary disease, lung cancer, cardiac failure, end-stage renal disease, and advanced neurological disease. There is no single intervention to relieve breathlessness, particularly chronic refractory breathlessness. This study identified that non-pharmacological treatments such as transcutaneous electrical nerve stimulation over acupoints, cognitive behavior therapy, inspiratory muscle training, and yoga were commonly used in breathlessness management and have shown potential benefits among older patient with breathlessness.

\section{Background}

$\mathrm{t}$ is too difficult to define breathlessness or dyspnea because of its subjective nature of experiencing breathing discomfort as a qualitatively distinct sensation that varies in intensity (American Thoracic Society, 1999, cited in Dunger et al. 2015). Patients report their breathlessness as the sensation of suffocation, choking or air hunger, an inability to take an adequate breath, and rapidity of breathing or increased effort related to breathing. It may also be an expression used to describe chest tightness (Cachia \& Ahmedzai, 2008; Loveridge \& White, 2012). Further, Thongkhamcharoen et al. (2012) and Ahmadi et al. (2014) described patients' experience in breathlessness in different terms such as weakness, tiredness, fatigue, feeling run-down, having insomnia, stress, or anxiety. Those expressions or feelings related to breathlessness are categorized into limited physical ability, psychological burdens, social life barriers, and environmental factors.

During the aging process, some changes occur in the respiratory system that affects the physiologic function of that system. Meiner (2013) points to two main changes in the respiratory system of older people, i.e. changes in the ribs and vertebrae and some changes in respiratory muscles, which both cause breathing difficulties. The ribs become less mobile and decrease the compliance of the chest wall. Osteoporosis and calcification of the costal cartilage affect the volume of the thorax. Some muscles which are involved in the respiration process also become weakened. The weakness makes breathing less efficient, so older adults use accessory muscles such as the abdominal, sternocleidomastoid, and trapezius for respiration (Rafii, Haghani \& Heidari 2017).

Because of this process, Cachia and Ahmedzai (2008) argued that to clearly understand the breathlessness in older people, health professionals should take aging process into account, because aging process can affect respiratory functions by reducing lung elasticity and respiratory muscle strength that ends in decreasing forced vital capacity, increasing air trapping, and impairing gas exchange. Also, it leads to a lower ventilatory response to hypoxia, hypercapnia, and higher ventilatory response to exercise at the same time.

Dyspnea is common in older people. Its reported prevalence varies from $20 \%$ to $60 \%$ (Ahmed, Steward \& O'Mahony, 2012); however, another study reported that the prevalence of dyspnea differs from $62 \%$ in older people (over 65 years) to $16 \%$ in men with the mean age of 59 years (Ho et al. 2001). It is also associated with poor functional status, reduced physical and mental health. Dyspnea is also common as a complaint presented by older people admitted to hospitals. Dyspnea in older people (aged $\geq 70$ years old) is significantly associated with mortality when age, gender, and underlying diseases are taken into account (Ahmed, Steward 
\& O’Mahony, 2012). About $60 \%$ of terminally-ill patients with cancer have experienced breathlessness, particularly in the last six weeks of their life (Ben-Aharon et al. 2008).

Furthermore, the prevalence of breathlessness in patients with lung cancer and advanced Chronic Obstructive Pulmonary Disease (COPD) ranges from $90 \%$ to $95 \%$ (Farquhar et al. 2014; Powell, 2014; Weingartner et al. 2015) while other cancers contribute to breathlessness around $50 \%$ to $70 \%$ (Farquhar et al. 2014; Weingärtner et al. 2015).

In addition, the prevalence of dyspnea is $88 \%$ in cardiac failure, $80 \%$ in end-stage renal disease, and $85 \%$ in advanced neurological disease. Both prevalence and intensity of breathlessness increase as death approaches (Powell, 2014). According to Simon et al. (2013), breathlessness is classified into two main categories; episodic breathlessness and continuous breathlessness. They can be differentiated by 24-h time as a preliminary cutoff period. They can be further subcategorized into triggered and non-triggered episodic breathlessness and short- and long-period continuous breathlessness. In contrast, Loveridge \& White (2012), and Yorke (2014b) classified breathlessness into three categories of acute, sub-acute or paroxysmal, and chronic breathlessness.

\section{Causes of breathlessness}

Breathlessness or dyspnea affects different patients to varying degrees (Barnett, 2009), and it is also common in the advanced stages of many diseases like COPD, heart failure, motor neuron disease and cancer (Ahamed \& Ahmedzai, 2011). It can also be present in other systemic conditions such as anemia, and neuromuscular weakness, or symptomatic conditions like pain, anxiety, and deconditioning (Loveridge $\&$ White, 2012). Finally, it can occur with other cancers and non-malignant diseases (Barnes, 2010).

There are some perspectives about the mechanism of breathlessness. According to the American Thoracic Society (1999), pathophysiological mechanisms of breathlessness are classified into four categories; reduction of ventilatory demand, lower ventilatory impedance, a decline of resistive load, and alteration of central perception (Thomas et al. 2011). Other scholars (Cachia \& Ahmedzai, 2008; Keenleyside \& Ahmedzai, 2008; Ahamed \& Ahmedzai, 2011) argued that there are three leading causes of dyspnea; increased chemical or neurological drive to breathe (e.g. by stimulation of chemoreceptors), increased work of breathing (e.g. in cardiac failure, pleural effusion), and decreased neuromuscular power (e.g. in Motor Neuron Disease [MND], cachexia).
Breathlessness in advanced disease is caused by a number of factors such as infection (pneumonia), cancer (affecting the lung primary or secondary), anemia, hypoxia/hypercapnia, anxiety/ panic, pain, pulmonary embolism, and pulmonary congestion; however, the particular contribution of each mechanism varies in each patient (Yorke, 2014a).

The common causes of dyspnea in patients with cancer are related to the direct effects of tumors. Some of these effects are obstruction, atelectasis, pleural effusion, superior vena cava syndrome, tracheal-esophageal fistula, and chest wall invasion. Dyspnea may also be a side effect of therapies like pulmonary fibrosis secondary to bleomycin or radiation, cardiomyopathy, acute dyspnea syndrome associated with Mitomycin-vinca alkaloid therapy, and therapeutic pneumonectomy. Anemia, obesity, infection, asthma, ascites, metabolic acidosis, pneumothorax, pulmonary emboli, heart failure, and acute anxiety may be some other causes of dyspnea (O'Rourke 2007; Cachia \& Ahmedzai 2008). Cryptogenic fibrosing alveolitis (Gysels, Bausewein \& Higginson, 2007) due to indoor air pollution, containing aldehydes and volatile organic compounds (VOCs), have also been mentioned as a cause of breathlessness (Bentayeb et al. 2013).

The gold standard for diagnosis of dyspnea is the patient's self-report. There is no other reliable, objective measure of the disorder. Measurements of respiratory rate, oxygen saturation, and arterial blood gases are not correlated with and do not confirm dyspnea. For example, patients may be hypoxemic but not dyspneic, or dyspneic but not hypoxemic (Thomas \& Von-Gunten, 2002). Chronic refractory breathlessness may be a daily experience for months or even years for some people with cardiorespiratory diseases (Currow, Higginson \& Johnson, 2013).

\section{Breathlessness management}

Breathlessness is a multifactorial problem caused by multiple mechanisms and thus lends itself to a range of interventions, each targeting a specific mechanism (Currow, Higginson \& Johnson, 2013). The management plan should ideally be directed to relieve or eliminate the underlying cause of the breathlessness like co-morbid condition, wherever this is feasible (Cachia \& Ahmedzai, 2008). In addition, Cachia and Ahmedzai (2008) argued that because of the troublesome side-effects (and costs) of drug or oxygen therapy, it was helpful to offer nonmedical approaches to the patient to alleviate dyspnea. Also, Currow, Higginson \& Johnson (2013) argued that there was no single intervention to relieve chronic refractory breathlessness, and holistic intervention was needed to provide incremental relief. 
Helping patients to reduce the sensation of experienced breathlessness is the main objective of any intervention in the management of breathlessness (Barnett, 2009). Nonpharmacological interventions require collaboration with patients using a range of strategies to complement pharmacological treatments for breathlessness (Cairns, 2012). There are three non-pharmacological approaches to breathlessness management; breathing, thinking; and functioning approaches, and each approach has various methods of implementation (Booth et al. 2014).

\section{Materials and Methods}

This review study used the (Preferred Reporting Items for Systematic Reviews and Meta-Analyses) PRISMA approach to select appropriate resources to determine nonpharmacological interventions for breathlessness management in older patients (Figure 1). Quantitative studies, including randomized placebo-controlled with pre-test and post-test design, retrospective and cohort studies, and pre- and post-intervention studies, were examined in this review. The search terms included "breathlessness", "dyspnea", "shortness of breath", "management", interventions", "therapy", "treatment", "non-pharmacological", "alternative therapies", "alternative medicine", "complementary therapies", "complementary medicine", "traditional medicine", "older people", and "older patient". The available title and abstract of the articles were reviewed systematically to find the relevant articles.

Two journal databases of CINAHL and MEDLINE were used in this study. Investigation of the relevant articles was performed by applying some criteria such as articles in English, published from 2008 to 2018, original paper, available in full-text, and peer-reviewed articles. The duplicated articles were removed to retrieve the proper articles of relevant abstracts. Data extraction was initiated by reviewing articles for their eligibility based on their titles and abstracts. Then, the extraction of the specific study characteristics started, including the study design, sample characteristics, type of intervention, outcomes, and intervention results.

The details of the extracted articles applying PRISMA were as follows: 1. Identification: We identified 188 articles in CINAHL and MEDLINE; 2. Screening: There were 170 articles after removing the duplicated articles $(n=18) ; 3$. Eligibility: Eligible articles in this study should meet the inclusion criteria of English articles, published after 2008, focused on non-pharmacological interventions, study samples older than 65 years people with dyspnea or breathlessness, and the original article.
The articles were excluded for several reasons such as title and abstract $(n=110)$, not related to the scope of this study $(n=40)$, being not academic journal such as review journals, letter to editor, short communication, case report and commentary $(n=4)$, not full text (abstract only) $(n=11)$.

\section{Included}

Articles which were included in this study should have met all the above-mentioned inclusion criteria. After the assessment, 5 articles were found as eligible and were analyzed.

\section{Results}

Based on the above criteria, 5 articles were selected (Table 1). Two studies focused on (Transcutaneous Electrical Nerve Simulation on acupoints) Acu-TENS in COPD patients (Lau \& Jones 2008; Liu et al. 2015), one study focused on cognitive behavior therapy in COPD patients (Howard et al. 2010), one study applied inspiratory muscle training in patients with thoracic malignancies (Liu et al. 2015), and the remaining study was on using yoga in lung cancer survivors (Molassiotis et al. 2015). This review focuses specifically on examining the influence of the following aspects of the study on intervention outcomes: study design, samples characteristics, intervention, and outcome and result of the intervention.

\section{Study design}

The majority of the studies were randomized controlled and conducted with pre-test and post-test design, and others were retrospective, cohort, and prospective studies. Two studies were conducted in 2015, one study in 2014, in 2010, and one in 2008 .

\section{Sample characteristics}

There were some important differences in sample characteristics between the studies, and a lack of information about the clinical aspects of samples in some reviewed studies was seen. For example, Lau and Jones (2008) study recruited 46 patients with stage I and II COPD according to the GOLD classification. Similarly, Howard et al. (2010) recruited 48 patients with COPD; however, there was no classification for those participants in terms of the stage of the disease.

In the study of Liu et al. (2015), 55 patients with stages I-IV of COPD based on the GOLD standard were recruited in the initial stage of the study, and 5 patients were excluded because FEV1\% predicted as $<20 \%$. In Foulad- 


\begin{tabular}{|c|c|}
\hline Identification & CINAHL \\
& MEDLINE \\
$(\mathrm{n}=188)$
\end{tabular}

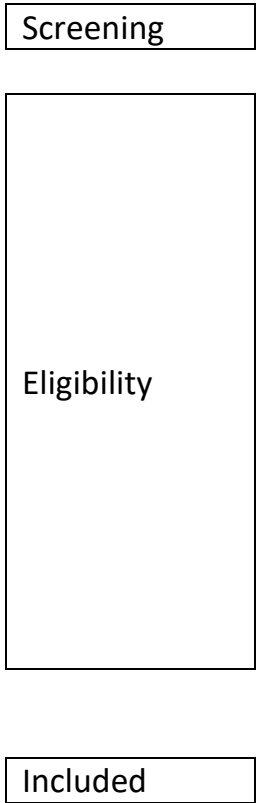

Included

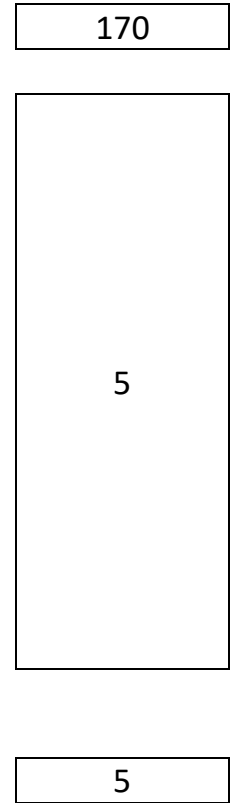

Duplicated articles $(n=18)$

Articles Excluded ( $n=165)$

- Title review and abstract $(n=110)$

- Not related to the scope of this study $(n=40)$

- Not academic articles such as review journal, letter to the editor, short communication, case report, and commentary $(n=$ 4)

- Not full text/abstract only $(n=11)$

Articles met criteria $(n=5)$
Figure 1. PRISMA analysis

bakhsh, Davis and Yarandi (2014) study, out of 9 patients who met the eligibility criteria, 3 men and 6 women were diagnosed with stages I-IIIa NSCLC (Non-small Cell Lung Carcinoma). While 46 patients with 70 NSCLC and advanced disease were recruited in Mollasiotis et al. (2015) study of them, 37 participants were men.

In the study by Howard et al. (2010), most participants were ex-smokers, which counted for around 85\% (34 participants), with an average of 39 pack years. While Lau and Jones classified the participants into three categories based on their smoking history; 15 patients were ex-smokers who stopped smoking for over 12 months, 22 patients were still smoker, and 9 patients were non-smokers. However, 9 ex-smoker participants (39) were in the experimental group, while $6(26 \%)$ participants were in the control group. In contrast, there were $3(13 \%)$ non-smokers in the experimental group, while there were $6(26 \%)$ non-smoker participants in the control group (2008).

In Liu et al. study, the smoking history in both intervention and control groups counted for around 22.80 and 22.20 years, respectively (2015). In Fouladbakhsh, Davis and Yarandi
Client- Centered Nursing Care

(2014) study, one participant reported a 40-year history of tobacco use, and the rest of the participants had not used tobacco and were not smokers. In contrast, there was no data about smoking history among participants in Mollasiotis et al. (2015) study. However, the participants who completed the questionnaires in T1, T2, and T3 (TNM staging) were about $91 \%, 85 \%$, and $81 \%$, respectively.

In the study by Howard et al. the participants would not be excluded if they had a co-morbid psychological diagnosis. However, based on the medical records, there was no participant with a formal diagnosis of clinical anxiety, panic disorder, or depression and some of the participants had comorbid conditions such as coronary heart disease and arthritis as well (2010). In Liu et al. (2015) study, some participants were excluded because they had a history of cardiovascular, endocrine, or neurologic disease, allergy to the addictive agents, pregnancy or lactation, upper respiratory tract disease, an acute exacerbation of COPD in the preceding 3 months, and participation in other clinical trials in the past 6 months (2015). 
In Fouladbakhsh, Davis and Yarandi (2014) study, one male participant withdrew from the study, and a female participant died unexpectedly before the start of yoga sessions. However, there was no information about the patient's comorbidities in both Lau and Jones and Mollasiotis et al. studies.

\section{Interventions}

The interventions have similarity in two studies (Lau \& Jones, 2008; Liu et al. 2015), although some important differences can be noted. In Lau and Jones, the studied participants in the experimental group received $45 \mathrm{~min}$ utes of Acu-TENS at bilateral acupoints Ex-B1 (known as Ding Chuan in Traditional Chinese Medicine), located at 0.5 'cun' lateral to the spinous process of $\mathrm{C} 7$, where 1 cun is the distance between the medial creases of the interphalangeal joints of the person's middle finger. The control group received 45 minutes of placebo-TENS.

A plastic film with no central pore was placed on the skin over each marked acupoint. The samples could see the output light flashing but not currently transmitted to the acupoint for 45 minutes. In Liu et al. study, 4 pairs of electrodes for TENS (6805-D model, Shantou City Medical Equipment Co. Ltd. Shantou City, China) were placed on the acupoints and fixed with adhesive tape. Treatment frequency was $2 \mathrm{~Hz}$, according to Franco et al. study. The electrodes of the same pair can only be connected to the same side to avoid direct contact across the body. In the control group (placebo Acu-TENS), the devices were set like the active treatment group, but no electrical current was applied. For both groups, the treatment was performed for 40 minutes, every 2 days, for 4 weeks (14 sessions).

In Howard et al. study, the patients in the intervention group were taught relaxation, breathing techniques, positioning, and effective chest clearance techniques. Then they received a handbook and a relaxation tape to continue the techniques and practice exercises daily. The groups were monitored by 6-week telephone follow-up. All groups received input from the same members of the multidisciplinary team, namely a respiratory CNS, health psychologist, physiotherapist, and occupational therapist. Groups ran consecutively for 12 months (8 groups in total, 6 participants on average per group).

Furthermore, in the Howard study, distraction techniques to modify behavioral response were key elements to reduce anxiety and panic. Howard results showed significant improvement in the participants' coping with breathlessness and ability to relax. Fouladbakhsh, Davis and Yarandi (2014) implemented yoga as an intervention. The yoga was applied for 8 consecutive weeks (40 minutes once per week) using a standardized protocol. Viniyoga, a form of hatha yoga, was selected for the protocol because of its focus on health concerns and modifiability according to individual limitations without compromising the overall effect.

A pressure threshold device was used to deliver (Inspiratory Muscle Training) IMT in Mollasiotis et al. study, which is commercially available by Phillips Respironics. When patients inhale through the IMT device, the valve obstructs the airflow until the patient creates enough inspiratory pressure to overcome the resistance developed by the spring-loaded valve. The patient must make the necessary inspiratory pressure, to open the valve and allow inhalation of air. The valve is calibrated and can be adjusted according to a percentage of the patient's maximum inspiratory pressure. The IMT protocol applied for 5 weekly sessions for 12 weeks, 30 $\mathrm{min} / \mathrm{d}$, divided over two sessions

Every study has different delivery methods for the intervention, in terms of who was in charge of delivering the program and how the program was delivered. Respiratory consultant or respiratory Clinical Nurse Specialist (CNS) delivered intervention programs in Howard et al. while in Lau and Jones study, the multidisciplinary team, namely a respiratory CNS, health psychologist, physiotherapist, and occupational therapist delivered those programs.

In Fouladbakhsh, Davis, and Yarandi study, the intervention was conducted by an experienced yoga teacher trained at the American Viniyoga Institute. The research team also was certified in advanced cardiopulmonary resuscitation to maximize participant's safety. The intervention of Acu-TENS in Liu et al. study was delivered by an acupuncturist. Interestingly, in the study conducted by Molassiotis, the inspiratory muscle training intervention was produced by the patients themselves after being educated on how to use the IMT device.

\section{Outcomes and result of the interventions}

In Lau and Jones study, the lung function measured as a forced expiratory volume in 1 second (FEV1) showed that after applying Acu-TENS to the intervention group, FEV1 significantly increased $10 \%$ more than that in the control group. Moreover, using a $100-\mathrm{mm}$ visual analog scale from 81 to 67 , those patients in the intervention group reported that their breathlessness has significantly decreased by 14 points more than that in the control 


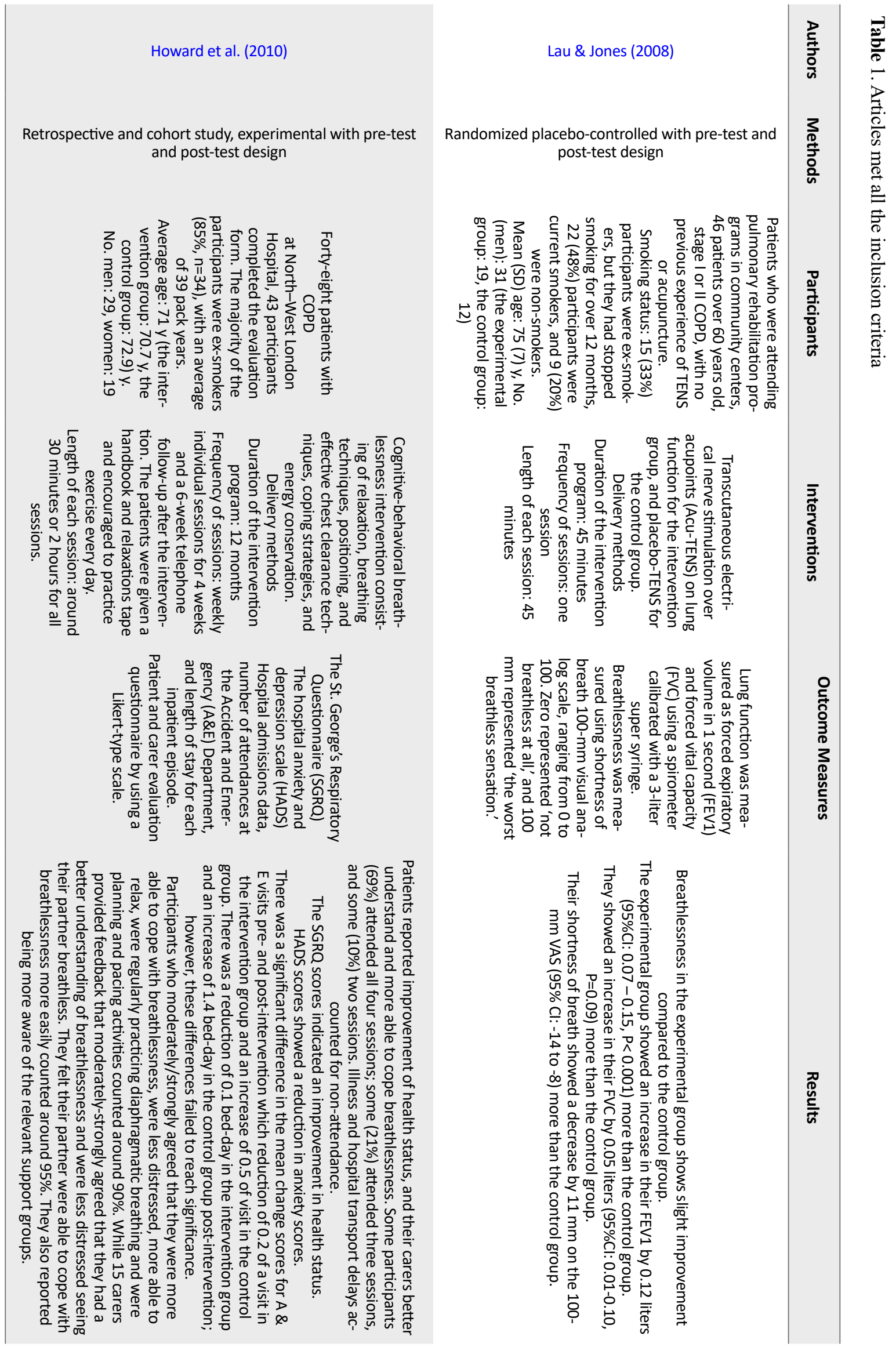




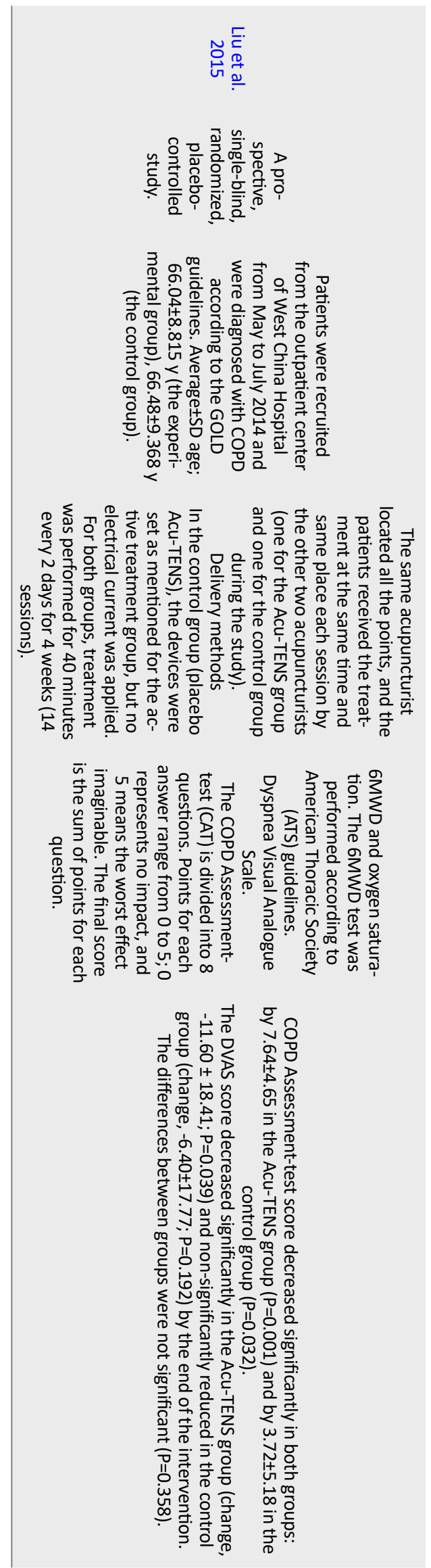

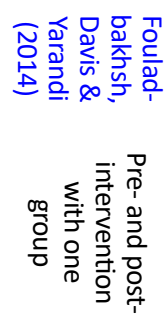
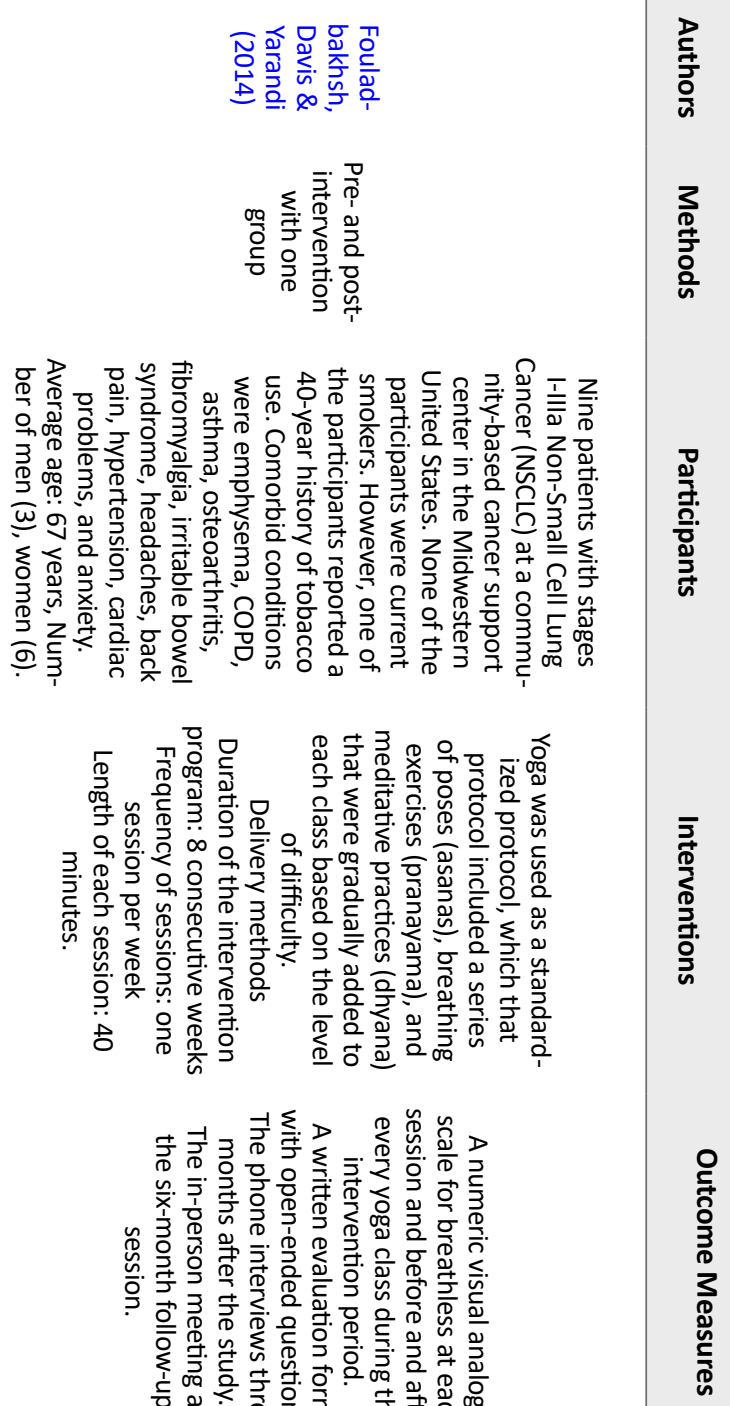

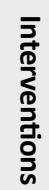
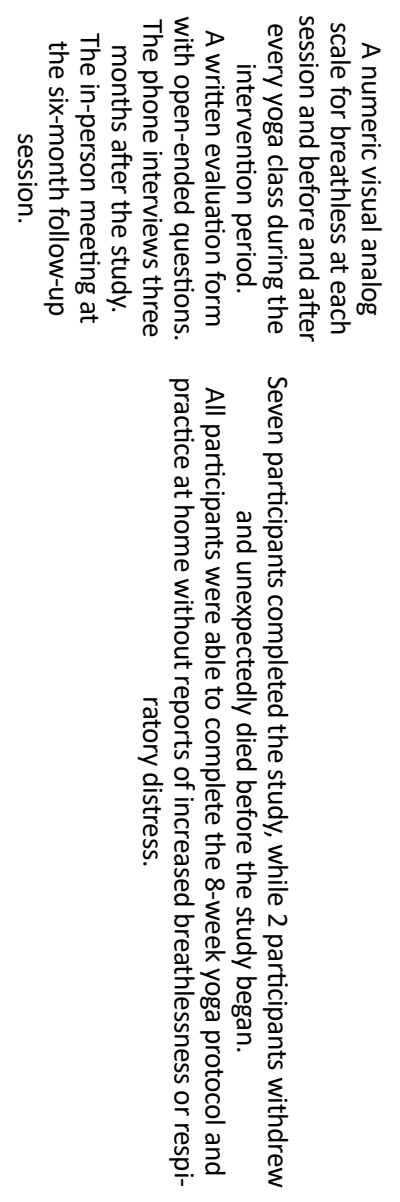

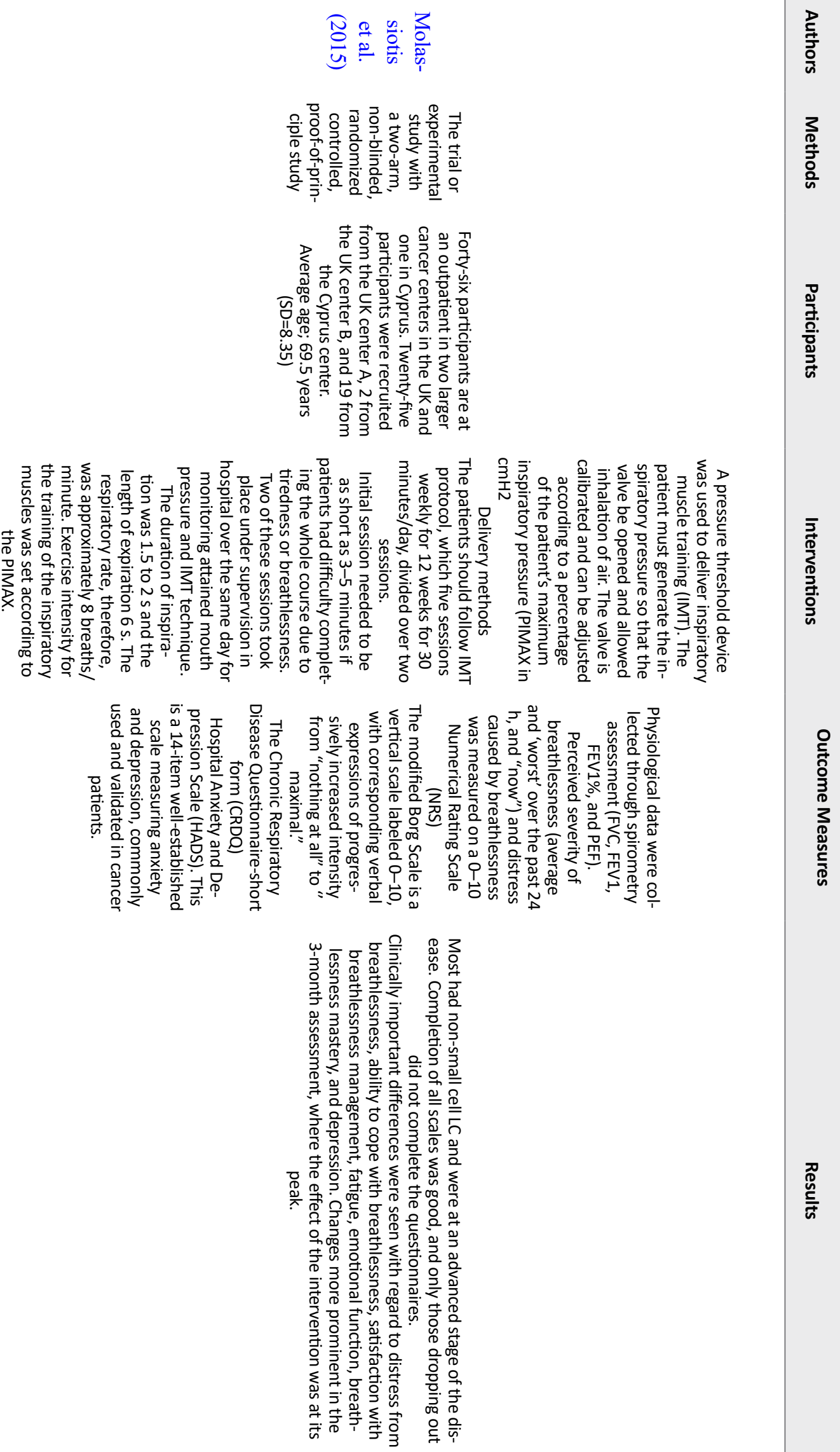
group. In Howard et al. study, the majority of patients were using salbutamol (85\%), with 65\% fluticasone/ salmeterol combination inhaler while $38 \%$ used tiotropium. Approximately one-third used Long-Term Oxygen Therapy (LTOT).

Furthermore, Howard et al. study measurement tool of breathlessness was the St. George's Respiratory Questionnaire (SGRQ). Total scores were significantly different over time (F3, 96=3.29, $\mathrm{P}<0.05, \mathrm{r}=0.2$ ). Pairwise comparisons after making a Bonferroni adjustment showed that the scores were significantly lower at the end, compared to the start of the intervention $(\mathrm{P}=0.005)$, indicating an improvement in reported health status. Howard et al. study results indicate that the overall health status and perceived impact of COPD on daily life have significantly improved. There were no significant differences in the SGRQ symptoms and activity domains. These differences were thus arguably neither clinically nor statistically significant. Similarly, Lau and Jones found that Acu-TENS improved FVC by only 3\% (50 mL) over placebo-TENS, which was statistically insignificant.

Liu et al. found a significant decrease in the COPD assessment-test both in Acu-TENS $(\mathrm{P}=0.001)$ and control groups ( $\mathrm{P}=0.032$ ). In contrast, when the (Dyspnea Visual Analog Scale) DVAS was used to measure the effect of Acu-TENS on dyspnea, the results showed that it was significantly different among both groups; in the AcuTENS group, the score decreased significantly while it just slightly decreased in the control group. Fouladbakhsh, Davis, and Yarandi found that some indicators such as sleep, mood, and quality of life significantly improved among the study participants. These indicators were measured by the Pittsburg Sleep Quality Index (PSQI), the profile of mood state brief and medical outcomes survey SF-36. The analysis showed their significance as $\mathrm{P}=0.002, \mathrm{P}=0.002$, and $\mathrm{P}=0.014$, respectively.

The breathlessness assessment would be used if the participant candidate could do yoga, and the protocol of yoga was modified during the intervention session to guarantee its safety. While it was reported in Molassiotis et al. study that there was no significant difference in mBorg Score (Modified Borg Score, a scale to measure dyspnea) among participants within IMT group, it was significantly different in the no-IMT group. Then, when the assessment results were compared in each time point between the two groups, it was found that the mBorg score of the no-IMT group was significantly higher than that of IMT group $(\mathrm{P}=0.029)$.

\section{Discussion}

Even though fan therapy (a cool draft of air from a handheld fan to reduce the feeling of breathlessness) has been commonly used to manage breathlessness among older patients (Bausewein 2010), among the reviewed articles, none of them emphasized and mentioned the application of fan intervention. Only four non-pharmacological interventions were identified and applied in these studies; Acu-TENS, cognitive-behavioral therapy, inspiratory muscle training, and yoga.

Lau and Jones found that FEV1 increased by 10\% (120 $\mathrm{mL})$ more after Acu-TENS than placebo-TENS. Also, Acu-TENS improved PVC by only 3\% (50 mL) higher than placebo-TENS which was statistically insignificant. However, Liu et al. in their study also found some differences in the outcomes of Acu-TENS when measured using the COPD assessment-test and DVAS. Even though the data on the effectiveness of acupuncture in patients with breathlessness are limited, Ozalevli (2013) argued that acupuncture might be an alternative approach to reduce breathlessness. Pan et al. (2000) in their randomized control trial study on COPD and cancer relatedbreathlessness patients found that acupuncture improved breathlessness symptoms and patients felt more relaxed even though 8 patients in that study were resistant to other treatments such as medicine and oxygen therapy. Another intervention which is similar to Acu-TENS is Neuromuscular Electrical Stimulation (NMES).

The NMES is delivered via a battery-operated device, which sends electrical impulses to muscles via electrodes on the skin. The impulses mimic the electrical signal received by muscles from the central nervous system and cause the muscles to contract (Lisy, White \& Pearson 2014). The NMES induces quadriceps contractions as an alternative to exercise; it is strong for COPD and not readily available for most patient groups (Powell 2014). However, the NMES program is just effective for patients with COPD in managing muscle weakness and improving exercise tolerance (Lisy, White \& Pearson 2014). Besides that, NMES was found to be a safe intervention and particularly suitable for those patients who were unable to participate in traditional exercise programs. Furthermore, based on patients' reports, their breathlessness and health-related quality of life had been improved significantly.

The effectiveness of yoga to relieve shortness of breath in patients with lung cancer has shown potential benefits. Besides, the yoga is a feasible option for lung cancer at varying stages, i.e. I to IIIa of the illness trajectory and 
may offer benefits for symptoms management and improving the quality of life. Yoga intervention is a holistic care treatment, consisting of coordination, movement, breathing, and relaxation, and mindfulness. Howard et al. study found that overall health status and perceived impact of COPD on daily life were significantly improved. According to patients' reports, most of the participants were more able to cope with breathlessness, less distressed, and more able to relax, when they were regularly practicing diaphragmatic breathing and planning pacing activities.

While the study carers reported that they had a better understanding of breathlessness and were less distressed seeing their patients' breathless and felt their patients were able to cope with breathlessness more easily. They also reported being more aware of relevant support groups. Similarly, Hately et al. (2003) argued that both the physical breathing techniques and psychological strategies were helpful. Those patients' improved physical and activity scores as measured by the Rotterdam subscale reflected the changes in the symptoms of breathlessness and functional capacity experienced by the patient group and demonstrated in the VASs.

Because of the strong relationship between dyspnea and cognitive and emotional aspects related to anxiety and depression, Cognitive Behavioral Therapy (CBT) intervention has been worthy of attention to some scholars. CBT is one of the methods of anxiety management that is widely used in clinical settings. CBT promotes self-mastery and delays the spiral of inactivity and deconditioning (Powel 2014). It has enormous potential for relieving the affective dimension of dyspnea (Norweg \& Collins 2013).

Furthermore, Thomas and Von-Gunten (2002) found out that patients who treated by using some methods such as breathing control, activity pacing, and relaxation techniques as a part of CBT show more improvement in dyspnea scores than patients treated with conventional approach without non-pharmacological therapies. Seamark, Seamark and Halpin (2007) study on anxiety and self-management behavior were successful in helping and improving asthma patients. They have implemented the intervention in patients with lung diseases such as COPD and lung cancer.

Webb, Moody and Mason (2000) believed that interventions such as relaxation could often be easily taught to patients and their caregivers, and may eliminate the problematic side effects associated with pharmacological therapies. They found, though, that few psychologi- cal interventions have been devised or tested to improve symptom management, functional status, or quality of life in patients with end-stage pulmonary disease. Energy conservation can offer the patient with disabling dyspnea to maintain such activities such as eating or grooming (Webb, Moody and Mason 2000). I agree with Barnett (2009) that patients should converse their energy to reduce their symptoms such as breathlessness, fatigue, anxiety, and panic, because, energy conservation techniques lead patients to do activities of daily living or exercise with useless energy. This technique also often included as a part of pulmonary rehabilitation (Leyshon, 2012).

Interestingly, Pan et al. (2000) found that patients who practiced progressive muscle relaxation for four weeks showed no significant improvement in dyspnea. However, the patients had less respiratory rate, heart rate, and anxiety at the end of the session, and their walking distance significantly improved compared to the control group. The study results by Connors, Graham \& Peel (2007) also reported as effective. However, these results are not statistically significant due to a small number of participants who completed the interventions, and that results may be questionable. Thus, taking the sample size into account to implement this intervention is needed because the authors acknowledged that the high dropout rate might affect the result of the study.

Although this review aimed to investigate the nonpharmacological interventions for breathlessness in older patients, the conclusion is not possible due to the lack of evidence in this field. However, this review reveals essential issues about non-pharmacological interventions for breathlessness, particularly in older patients.

According to the findings of this review, non-pharmacological interventions for older patients with breathlessness differ based on the researcher's interest and patient's disease. Some interventions were identified in this review, such as Acu-TENS, cognitive behavioral therapy, inspiratory muscle training, and yoga. However, co-morbidities were important issues in this review. Another issue in those studies was that the number of participants who completed the interventions was small. Also, though these non-pharmacological interventions have the potential to improve patients' dyspnea, it seems that smoking history should have been included in the recruitment criteria. 


\section{Ethical Considerations}

\section{Compliance with ethical guidelines}

There was no ethical principles to be considered in this research.

\section{Funding}

This research did not receive any specific grant from funding agencies in the public, commercial, or not-forprofit sectors.

\section{Conflict of interest}

The author declare no conflicts of interests.

\section{References}

Ahamed, A. \& Ahmedzai, S. H., 2011. Breathlessness in advanced disease. Medicine, 39(11), pp. 651-5. [DOI:10.1016/j. mpmed.2011.08.015]

Ahmadi, N.S., et al., 2014. Breathlessness in everyday life from a patient perspective: A qualitative study using diaries. Palliative $\mathcal{E}$ Supportive Care, 12(3), pp. 189-94. [DOI:10.1017/ S1478951512001095] [PMID]

Ahmed, T., Steward, J. A. \& O'Mahony, M. S., 2012. Dyspnoea and mortality in older people in the community: A 10-year follow-up. Age and Ageing, 41(4), pp. 545-9. [DOI:10.1093/ageing/afs049] [PMID]

Barnes, D., 2010. Breathlessness in advanced disease. 1: Definitions, epidemiology and pathophysiology. Nursing Times, 106(43), pp. 20-1. [PMID]

Barnett, M., 2009. Management of breathlessness. Journal of Community Nursing, 23(12), pp. 6-13.

Bausewein, C., et al., 2010. Effectiveness of a hand-held fan for breathlessness: A randomised phase II trial. BMC Palliative Care, 9, p. 22. [DOI:10.1186/1472-684X-9-22] [PMID] [PMCID]

Ben-Aharon, I., et al., 2008. Interventions for alleviating cancerrelated dyspnea: A systematic review. Journal of Clinical Oncology, 26(14), pp. 2396-404. [DOI:10.1200/JCO.2007.15.5796] [PMID]

Bentayeb, M., et al., 2013. Higher prevalence of breathlessness in elderly exposed to indoor aldehydes and VOCs in a representative sample of French dwellings. Respiratory Medicine, 107(10), pp. 1598-607. [DOI:10.1016/j.rmed.2013.07.015] [PMID]

Boland, J., et al., 2013. Palliative care for people with nonmalignant lung disease: Summary of current evidence and future direction. Palliative Medicine, 27(9), pp. 811-6. [DOI:10.1177/0269216313493467] [PMID]

Booth, S., et al., 2014. Positions to ease breathlessness. In S. Booth, J. Burkin, C. Moffat, A. (eds), Spathis, Managing Breath- lessness in Clinical Practice. Berlin: Springer. [DOI:10.1007/978 1-4471-4754-1]

Cachia, E. \& Ahmedzai, S. H., 2008. Breathlessness in cancer patients. European Journal of Cancer, 44(8), pp. 1116-23. [DOI:10.1016/j.ejca.2008.03.004] [PMID]

Cairns, L., 2012. Managing breathlessness in patients with lung cancer. Nursing Standard, 27(13), pp. 44-49. [DOI:10.7748/ ns2012.11.27.13.44.c9450] [PMID]

Connors, S., Graham, S. \& Peel, T., 2007. An evaluation of a physiotherapy led non-pharmacological breathlessness programme for patients with intrathoracic malignancy. Palliative Medicine, 21(4), pp. 285-7. [DOI:10.1177/0269216307079172] [PMID]

Currow, D., et al., 2013. Evidence-based intervention for chronic refractory breathlessness: practical therapies that make a difference. British Journal of General Practice, 63(616), pp. 609-10 [DOI:10.3399/bjgp13X674611] [PMID] [PMCID]

Currow, D. C., Higginson, I. J. \& Johnson, M. J., 2013. Breathlessness- current and emerging mechanisms, measurement and management: A discussion from an European Association of Palliative Care workshop. Palliative Medicine, 27(10), pp. 932-8. [DOI:10.1177/0269216313493819] [PMID]

Dunger, C., et al., 2015. Breathlessness and crises in the context of advanced illness: A comparison between COPD and lung cancer patients. Palliative E Supportive Care, 13(2), pp. 229-37. [DOI:10.1017/S147895151300120X] [PMID]

Farquhar, M., et al., 2014. Is a specialist breathlessness service more effective and cost-effective for patients with advanced cancer and their carers than standard care? Findings of a mixed-method randomised controlled trial. BMC Medicine, 12, p. 194. [DOI:10.1186/PREACCEPT-1197669166131735] [PMID] [PMCID]

Meiner, S. E., 2013. Gerontologic nursing-e-book. London: Elsevier Health Sciences.

Fouladbakhsh, J. M., Davis, J. E., \& Yarandi, H. N., 2014. A pilot study of the feasibility and outcomes of yoga for lung cancer survivors. Oncology Nursing Forum, 41(2), pp. 162-74. [DOI:10.1188/14.ONF.162-174] [PMID]

Gysels, M., Bausewein, C., \& Higginson, I., 2007. Experiences of breathlessness: A systematic review of the qualitative literature. Palliative Supportive Care, 5(3), pp. 281-302. [DOI:10.1017/ S1478951507000454]

Hately, J., et al., 2003. Breathlessness clinics within specialist palliative care settings can improve the quality of life and functional capacity of patients with lung cancer. Palliative Medicine 17(5), pp. 410-7. [DOI:10.1191/0269216303pm752oa] [PMID]

Ho, S., et al., 2001. Dyspnoea and quality of life in older people at home. Age and Ageing, 30(2), pp. 155-9. [DOI:10.1093/ageing/30.2.155] [PMID]

Howard, C., et al., 2010. The effectiveness of a group cognitive-behavioural breathlessness intervention on health status, mood and hospital admissions in elderly patients with chronic obstructive pulmonary disease. Psychology, Health $\mathcal{E}$ Medicine, 15(4), pp. 371-85. [DOI:10.1080/13548506.2010.4821 42] [PMID] 
Keenleyside, G., \& Ahmedzai, S. H., 2008. Breathlessness in advanced disease. Medicine, 36(2), pp. 82-7. [DOI:10.1016/j. mpmed.2007.12.010]

Lau, K. S. \& Jones, A. Y., 2008. A single session of Acu-TENS increases FEV1 and reduces dyspnoea in patients with chronic obstructive pulmonary disease: A randomised, placebo-controlled trial. Australian Journal of Physiotherapy, 54(3), pp. 17984. [DOI:10.1016/S0004-9514(08)70024-2]

Leyshon, J., 2012. Managing severe breathlessness in patients with end-stage COPD. Nursing Standard, 27(6), pp. 48-56. [DOI:10.7748/ns2012.10.27.6.48.c9349] [PMID]

Lisy, K., White, H., \& Pearson, A., 2014. Overview of reviews: Mechanical interventions for the treatment and management of chronic obstructive pulmonary disease. International Journal of Nursing Practice, 20(6), pp. 701-8. [DOI:10.1111/ijn.12303] [PMID]

Liu, X., et al., 2015. Effects of transcutaneous electrical acupoint stimulation on patients with stable chronic obstructive pulmonary disease; A prospective, single-blind, randomized, placebo-controlled study. The Journal of Alternative and Complementary Medicine, 21(10), pp. 610-6. [DOI:10.1089/acm.2014.0284] [PMID]

Loveridge, J., \& White, V., 2012. Breathlessness and cough. Medicine, 41(2), pp. 108-12. [DOI:10.1016/j.mpmed.2012.11.009]

Molassiotis, A., et al., 2015. The effect of resistance inspiratory muscle training in the management of breathlessness in patients with thoracic malignancies; A feasibility randomized trial. Support Care Cancer, 23(6), pp. 1637-45. [DOI:10.1007/ s00520-014-2511-x] [PMID]

Norweg, A. \& Collins, E. G., 2013. Evidence for cognitive- behavioral strategies improving dyspnea and related distress in COPD. International Journal of Chronic Obstructive Pulmonary Disease, 8, p. 439-51. [DOI:10.2147/COPD.S30145] [PMID] [PMCID]

O'Rourke, M., 2007. Clinical dilemma: Dyspnea. Seminars in Oncology Nursing, 23(3), pp. 225-31. [DOI:10.1016/j. soncn.2007.05.007] [PMID]

Ozalevli, S., 2013. Impact of physiotherapy on patients with advanced lung cancer. Chronic Respiratory Disease, 10(4), pp. 22332. [DOI:10.1177/1479972313508965] [PMID]

Pan, C.X., et al., 2000. Complementary and alternative medicine in the management of pain, dyspnea, and nausea and vomiting near the end of life: A systematic review. Journal of Pain and Symptom Management, 20(5), pp. 374-87. [DOI:10.1016/S08853924(00)00190-1]

Powell, B., 2014. Managing breathlessness in advanced disease. Clinical Medicine, 14(3), pp. 308-11. [DOI:10.7861/clinmedicine.14-3-308] [PMID] [PMCID]

Rafii, F., Haghani, H. \& Heidari B. F., 2017. [Health-related quality of life and related factors in patients with chronic obstructive pulmonary disease (Persian)]. Journal of Client Centred Nursing Care, 3(1), pp. 45-50. [DOI:10.32598/jccnc.3.1.45]

Seamark, D. A., Seamark, C. J., \& Halpin, D. M. 2007. Palliative care in chronic obstructive pulmonary disease: A review for clinicians. Journal of the Royal Society of Medicine, 100(5), pp. 225-33. [DOI:10.1258/jrsm.100.5.225] [PMID] [PMCID]
Simon, S.T., et al., 2013. Episodic and continuous breathlessness: a new categorization of breathlessness. Journal of Pain and Symptom Management, 45(6), pp. 1019-29. [DOI:10.1016/j. jpainsymman.2012.06.008] [PMID]

Thomas, J. R, \& Von-Gunten, C. F., 2002. Clinical management of dyspnea. Lancet Oncology, 3(4), pp. 223-8. [DOI:10.1016/ S1470-2045(02)00713-1]

Thomas, S., et al., 2011. Breathlessness in cancer patients-implications, management and challenges. European Journal of Oncology Nursing, 15(5), pp. 459-69. [DOI:10.1016/j.ejon.2010.11.013] [PMID]

Thongkhamcharoen, R., et al., 2012. Dyspnea management in Palliative home care: A case series in Malaysia. Indian Journal of Palliative Care, 18(2), pp. 128-33. [DOI:10.4103/09731075.100835] [PMID] [PMCID]

Webb, M., Moody, L. E. \& Mason, L. A., 2000. Dyspnea assessment and management in hospice patients with pulmonary disorders. American Journal of Hospice and Palliative Medicine 17(4), pp. 259-64. [DOI:10.1177/104990910001700412] [PMID]

Weingärtner, V., et al., 2015. Characteristics of episodic breathlessness as reported by patients with advanced chronic obstructive pulmonary disease and lung cancer: Results of a descriptive cohort study. Palliative Medicine, 29(5), pp. 420-8. [DOI:10.1177/0269216314563428] [PMID]

Yorke, J., 2014 a. Breathlessness in the community: Part 1 - assessment. Journal of Community Nursing, 28(5), pp. 83-90.

Yorke, J., 2014 b. Breathlessness in the community: Part 2 - management. Journal of Community Nursing, 28(6), pp. 44-51. 
This Page Intentionally Left Blank 